\title{
Survival analysis in out-of-hospital cardiac arrest patients with shockable rhythm directly transport to Heart Centers
}

\author{
Chung-Cheng Yeh ${ }^{1,2, \dagger}$, Chia-Hau Chang ${ }^{1,2,3, \dagger}$, Chen-June Seak ${ }^{1,3}$, Chen-Bin Chen ${ }^{1,3}$, \\ Yi-Ming Weng ${ }^{1,4}$, Chi-Chun Lin ${ }^{1,5}$, Chien-Hsiung Huang ${ }^{1}$, Hsiao-Jung Tseng ${ }^{6}$, \\ Chip-Jin Ng${ }^{1}$, Li-Heng Tsai ${ }^{1, *}$
}

\author{
${ }^{1}$ Department of Emergency Medicine, \\ Chang Gung Memorial Hospital, Linkou \\ and College of Medicine, Chang Gung \\ University, 333 Tao-Yuan, Taiwan \\ ${ }^{2}$ Department of Emergency Medicine, \\ Chang Gung Memorial Hospital, \\ Keelung Branch, Taiwan \\ ${ }^{3}$ Department of Emergency Medicine, \\ New Taipei Municipal Tucheng Hospital, \\ 236 New Taipei City, Taiwan \\ ${ }^{4}$ Department of Emergency Medicine, \\ Taoyuan General Hospital, Ministry of \\ Health and Welfare, 33004 Taoyuan, \\ Taiwan \\ ${ }^{5}$ Department of Emergency Medicine, \\ Ton-Yen General Hospital, 302 Zhubei, \\ Taiwan \\ ${ }^{6}$ Biostatistical Unit, Clinical Trial Center, \\ Chang Gung Memorial Hospital, 333 \\ Linkou, Taiwan

\section{*Correspondence \\ lihan0509@gmail.com \\ (Li-Heng Tsai)}

$\dagger$ These authors contributed equally.

\begin{abstract}
Background: This study aimed to evaluate whether out-of-hospital cardiac arrest (OHCA) patients with initial shockable rhythm without prehospital return of spontaneous circulation (ROSC) who are directly transported to Heart Centers in appropriate time will have better post-cardiac arrest four months survival and neurological outcomes at discharge.

Methods: This retrospective study assessed the data of 1,588 OHCA patients with shockable rhythm and without prehospital ROSC collected from the registry database of Taoyuan City between January 2014 and June 2018. The relationships of transport time to Heart Centers with survival at discharge and with neurological outcomes were investigated for survival analysis.

Results: Among the 1,588 OHCA patients with initial shockable rhythm and without prehospital ROSC, 1,222 (77.0\%) and $366(23.0 \%)$ were transported to Heart Centers and non-Heart Centers, respectively. However, the transport to Heart Centers was associated with an increased survival at discharge (adjusted odds ratio [aOR] 2.00, 95\% confidence interval [CI], 1.42-2.81) and good neurological outcomes (cerebral performance category [CPC] 1 and 2) (aOR 3.14, 95\% CI, 1.88-5.23), regardless of the transport time. The overall mortality reduction for Heart Centers was $39 \%$ (hazard ratio $[\mathrm{HR}]=0.61 ; 95 \%$ CI $0.47-0.78$ ), compared to that for non-Heart Centers. At 120 days of follow-up, the results showed a higher survival rate for patients who were transported to Heart Centers within a short time. The percentages of good CPC showed a better distribution for non-Heart Centers versus those for Heart Centers.

Conclusions: Adult OHCA patients with initial shockable rhythm and without prehospital ROSC who were transported to Heart Centers directly had better post-cardiac arrest survival and good neurologic outcomes, regardless of the transport time.
\end{abstract}

\section{Keywords}

Out-of-hospital cardiac arrest; Heart Centers; Transport time; Survival at discharge

\section{Introduction}

Out-of-hospital cardiac arrest (OHCA) remains a global health issue that leads to high mortality and poor neurologic outcomes. Patient survival outcomes depend on the characteristics of different target hospitals, regions, and nations. Emergency medical services (EMSs) also play an important role in patient survival. The survival rate ranges from $3.0 \%$ to $16.3 \%$, with a median of $8.4 \%$, in North America [1]; $1.9 \%$ to $3.1 \%$ in Japan [2]; and $1 \%$ in Taiwan [3]. In Vienna, survival analysis suggested a $53 \%$ decrease in risk with initial shockable rhythm compared to that with non-shockable rhythm [4]. It is difficult to follow a consistent protocol because there are differences in the characteristics of OHCA patients between regions.
For OHCA patients, early initiation of the chain of survival is important. A strong chain of survival can improve the chances of survival and recovery for victims of cardiac arrest [5-8]. In the chain of survival, early defibrillation is a strong predictor of survival in OHCA patients with shockable rhythm [9]. Shockable rhythm is known to have a higher survival rate and higher association with acute coronary syndrome than non-shockable rhythm, with better chance of return of spontaneous circulation (ROSC) [10]. Among OHCA patients who were transported to the hospital by emergency medical technicians (EMTs), initial shockable rhythms accounted for approximately $22.9 \%$ of cases in the USA and $18.8 \%$ of cases in Taiwan in the control group [1, 11]. In addition, there was a higher incidence of acute coronary lesions in 
patients with shockable rhythm than in those without shockable rhythm (29.7\% vs. $16.4 \%)$ in Spain, leading to a higher need for primary percutaneous coronary intervention (PCIs) [12]. Moreover, the last cycle of the survival chain, i.e., postcardiac arrest care treatment protocol, including immediate PCI and target temperature management (TTM), also plays an important role in OHCA patients' survival and neurologic outcomes. Therefore, considering the aforementioned reasons, whether or not the target hospital could perform PCI, TTM, or extracorporeal cardiopulmonary resuscitation (E-CPR) makes a big difference. Another study suggested that directly sending OHCA patients to hospitals that can perform definitive treatment would result in better outcomes [13].

Heart Centers, also called as Heart Centers, high-case volume centers, critical care medical centers, or tertiary care centers, should have the ability to not only perform 24/7 primary PCIs but provide high-quality post-resuscitation care including TTM in intensive care facilities, high level of specialization, and high volume of post-resuscitation care patients [14-16]. More evidence suggested that direct transport of OHCA patients to Heart Centers led to better survival at discharge and good neurological outcomes [17, 18]. Kragholm et al. [19] reported no differences in the increased survival rate of different transport times in patients "with" prehospital ROSC. However, whether transport time affects the prognosis or the safety of bypassing the nearest hospitals to Heart centers of OHCA patients with initial shockable rhythm and without prehospital ROSC has not been largely discussed [13].

In previous concepts, heart disease is reported to be responsible for the majority of OHCA cases, especially those with shockable rhythms. The short-term prognosis of OHCA patients after discharge from Heart centers is unknow. Therefore, the aim of this study was to evaluate whether OHCA patients with initial shockable rhythm and without prehospital ROSC should be directly transported to Heart Centers even under longer transport time, and its effect on post-cardiac arrest survival outcomes after discharge.

\section{Material and methods}

\subsection{Study design and setting}

This was a retrospective cohort study conducted between January 2014 and June 2018 at Taoyuan City in northern Taiwan. The OHCA database has prospectively collected data and is based on the Utstein reporting template. All hospital emergency departments (EDs) and EMSs in Taoyuan City (population 2,132,854; area 1,221 $\mathrm{km}^{2}$ ), Taiwan, mandatorily reported data to the database. The database includes the data of 35 EMSs including 13 first-aid hospitals. The collected information included patient characteristics, EMS parameters, and clinical outcomes from the EMS run sheets and hospital medical records. The Taoyuan EMT followed the same protocol and delivered OHCA patients to the closest hospital and did not support bypass to the Heart Center. The authors selected the Heart Centers if the following criteria were met: (a) ability to start up 24/7 PCI in emergency department and perform PCI by cardiologist if elevated ST segment of electrocardiography (ECG) was found or highly suspected for cardiac etiology; (b) could perform TTM in the ED or intensive care unit (ICU) if the OHCA patients were still comatose status and negative finding in brain computed tomography (CT); (c) has a 24/7 cardiovascular surgeon consultant team after the failure of PCI or cardiologist suggestion; and (d) has an integrated ED and ICU for post-cardiac arrest care (including maintain mean arterial pressure (MAP) $>65 \mathrm{mmHg}$ and respiratory parameters). This study was approved by the Hospital Ethics Committee on Human Research of the Chang Gung Medical Foundation (Taiwan, ROC; IRB Permit No.: 201600635B0).

\subsection{Patient involvement}

We use the database of the Taoyuan's EMS station, data obtained which entered research design and analysis are all patient and public information removed.

\subsection{Data collection}

The OHCA database collected information on patient characteristics (age, sex, location of cardiac arrest, witness status, automated external defibrillator (AED) record, and bystander CPR), EMS parameters (response time, scene time interval [STI]), transport time, level of EMTs, number of EMT, type of life support (management by EMTs at the scene), hospital characteristics (CPR protocol, TTM protocol, and OHCA analysis meeting), and clinical outcomes (survival at discharge, discharge neurological outcome based on cerebral performance category [CPC], which were assessed by the attending physician, and 4 months' survival by phone contact to family) from the EMS run sheets and hospital medical records.

\subsection{Study population}

We used data from the OHCA database for the period 20142018, reported by 13 first-aid hospitals in Taoyuan City: 8 Heart Centers and 5 non-Heart Centers.

The inclusion criteria were age older than 20 years and initial shockable rhythm at the scene by AED without prehospital ROSC. The exclusion criteria were age younger than 20 years, families' refusal to send the patients to hospitals or signed DO NOT RESCUSCITATE (DNR), pronounced death at the scene, etiology with obvious non-cardiac etiology (trauma or intoxication), missing data (no AED record and EMS record), non-shockable rhythm.

\subsection{Outcome measures}

Taoyuan's EMS index for OHCA records 2-hour survival for OHCA patients and the definition was recorded survival time after ROSC. Hence, the primary outcome was survival over 2 hours after ROSC and 4-month survival after discharge. The secondary outcomes were admission to the ICU, survival at discharge and good neurological outcomes at discharge using the CPC scale (from 1 to 5). The CPC scale was evaluated by the attending physician. CPC 1 refers to good cerebral performance; 2 , moderate cerebral disability; 3 , severe cerebral disability; 4 , coma or vegetative state; and 5, death. CPC scores of 1 or 2 were defined as good CPC scores. 


\subsection{Taoyuan emergency medical technicians}

EMTs play an important role in prehospital medical care. In 2018, 58 EMT-1 (EMT-basic), 921 EMT-2 (EMT intermediate), and 123 EMT-P (EMT-Paramedic) were employed in Taoyuan's EMS stations. In Taiwan, EMT-Ps can perform only endotracheal intubation and administer epinephrine and amiodarone parenterally according to Taiwan's laws. EMT$2 \mathrm{~s}$ were certified to use a laryngeal mask airway and set intravenous lines for fluid resuscitation as well as in EMTPs. All grades of EMTs were trained and could operate the AEDs. It is necessary for the least EMT-intermediate to attend during OHCA. The mechanical CPR device was equipped with LUCAS and Autopulse on the ambulance.

\subsection{Definition of EMS parameters}

The interval from calling 119 to the arrival of an ambulance at the scene was defined as the response time. STI was defined as the time the EMS remained at the scene. We estimated the time taken by an ambulance from leaving the scene to arrival at the first-aid hospital and defined this as the transport time.

\subsection{Statistical analysis}

Results of descriptive analysis of patient characteristics by the destination hospital are shown in Table 1. Continuous variables were presented as mean and standard deviation. Categorical variables were presented as frequencies and proportions. Two-sample independent $t$-tests and Chi-square tests were used for continuous variables and categorical variables, respectively, to examine the differences between groups.

Owing to the fact that patient assignment was not a random process, to avoid potential selection bias, which might be caused by the type of hospital to which patients are delivered, we used propensity scores to overcome this problem. Variables observed before delivery were used to estimate the tendency to assign patients to Heart Centers or non-Heart Centers. A logistic regression model was used to estimate the probability as a propensity score with 10 variables including age, sex, location of arrest, witness of collapse, provision of bystander, shift of non-shock rhythm, number of EMTs, type of life support, response time, and STI. The estimated probability (score) was seen as the propensity score and was categorized into quintiles. A cutoff was determined 7.16 (minutes) by the average probability of survival at discharge corresponding to the critical point of the transport time using generalized additive modeling technique by R3.6.0 (Supplementary Fig. 1). Transport time larger than 7.16 minutes was defined as a long transport time.

We used multivariable conditional logistic regression models with quintiles of propensity score as stratified intercept to examine the effect of hospital type and length of transport time on prognostic binary outcomes including survival over two hours since admission to the ICU, and, consequently, alive to discharge. For secondary analysis, we analyzed the data of patients who were still alive after 2 hours. We used the Cox proportional hazards model to evaluate the hazard ratios (HRs). The Kaplan-Meier method was used to construct the survival curve for different situations combined with patients' trans- portation to the Heart Center and time consumption. A logrank test was used to confirm differences in survival curves. Statistical analyses were performed using IBM SPSS (Version 25.0. Released 2017; IBM Corp., Armonk, NY) and R with generalized additive model package. $p<0.05$ was considered to indicate statistical significance.

\section{Results}

There were 10,437 cases of cardiac arrest recorded in the OHCA database between January 2014 and December 2018. Patients were excluded due to refusal by family to go to the hospital $(1,235 / 10,437 ; 11.8 \%)$, pronounced death at the scene $(984 / 10,437 ; 9.4 \%)$, non-shockable rhythm $(4,763 / 10,437 ; 45.6 \%)$, trauma $(1,432 / 10,437$; $13.7 \%)$, intoxication $(15 / 10,437 ; 0.14 \%)$, age under 20 years $(272 / 10,437 ; 2.6 \%)$, missing data $(67 / 10,437 ; 0.64 \%)$, and pre-hospital ROSC $(81 / 10,437 ; 0.78 \%)$. Finally, we enrolled $1,588(15.2 \%)$ patients in this study (Fig. 1).

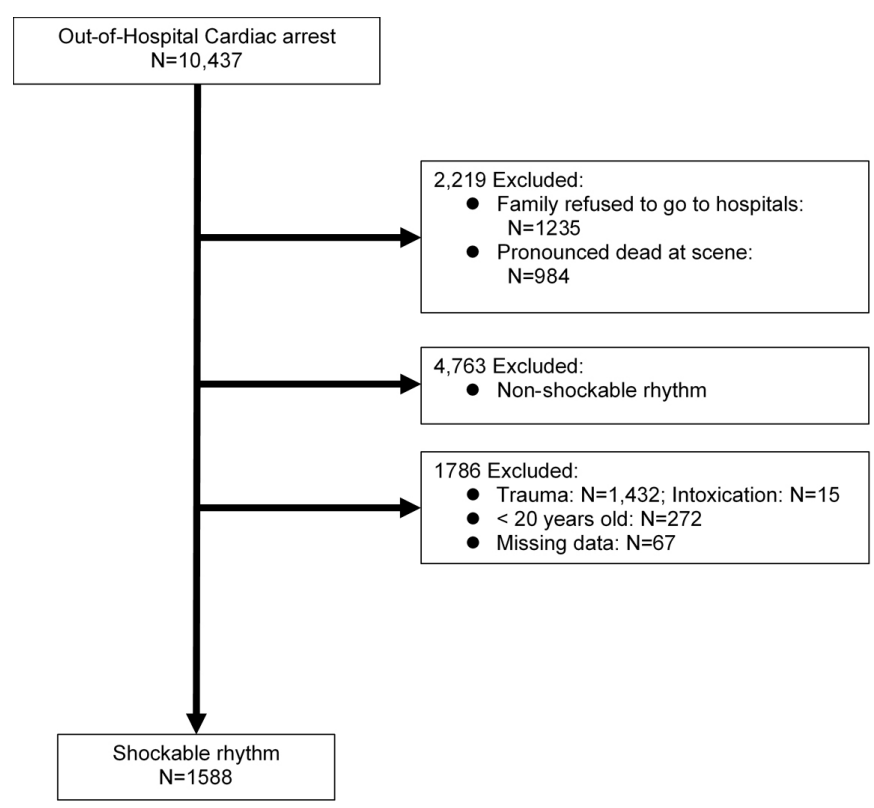

F I G U R E 1. Flow Diagram of patient enrollment.

Abbreviations: ROSC, return of spontaneous circulation.

Table 1 shows the characteristics of patients by the Heart Center. The Heart Centers received 1,222 patients (77\%) and the non-Heart Centers received 366 patients $(23 \%)$. There were no significant differences in patient characteristics including age, sex, location of cardiac arrest, witness when the patient collapsed, shifting to non-shockable rhythm, number of EMTs, and type of life support between the Heart Center and the non-Heart Center. Patients delivered to the Heart Center had more bystander CPR $(p=0.047)$, higher EMT level ( $p$ $=0.027)$, longer mean transport time $(p<0.001)$, and better outcomes including survival over 2 hours $(p<0.001)$, survival at discharge $(p<0.001)$, admission to the ICU $(p=0.001)$, and better CPC score $(p<0.001)$ The post-cardiac arrest cares of Heart Center in emergency PCI and TTM were 27.2\% and $33.7 \%$, respectively.

Results of multivariable conditional logistic regression models with quintile propensity score stratification are shown in 
TA B L E 1. Characteristics by destination hospital.

\begin{tabular}{|c|c|c|c|c|}
\hline \multirow[b]{2}{*}{ Variables } & \multirow{2}{*}{$\begin{array}{l}\text { Total } \\
(\mathrm{n}=1588)\end{array}$} & \multicolumn{2}{|c|}{ Heart Center } & \multirow[t]{2}{*}{$p$-value } \\
\hline & & No $(n=366)$ & Yes $(n=1222)$ & \\
\hline Age, years, mean (SD) & $62.19(15.82)$ & $61.95(16.19)$ & $62.61(15.67)$ & 0.4968 \\
\hline Sex $=$ Male & $1267(79.8 \%)$ & $288(78.7 \%)$ & $926(80.1 \%)$ & 0.551 \\
\hline Location of arrest & & & & 0.064 \\
\hline Public & $552(34.8 \%)$ & $142(38.8 \%)$ & $410(33.6 \%)$ & \\
\hline Residential & $1036(65.2 \%)$ & $224(61.2 \%)$ & $812(66.4 \%)$ & \\
\hline Witness collapse & $1004(63.2 \%)$ & $217(59.3 \%)$ & $787(64.4 \%)$ & 0.075 \\
\hline Provision of bystander CPR & $678(42.8 \%)$ & $140(38.3 \%)$ & $539(44.1 \%)$ & 0.047 \\
\hline Shift to non-shock rhythm & $939(59.1 \%)$ & $203(55.5 \%)$ & $736(60.2 \%)$ & 0.104 \\
\hline No. of EMTs & & & & 0.061 \\
\hline 2 & $879(55.4 \%)$ & $210(57.4 \%)$ & $669(54.7 \%)$ & \\
\hline $3-4$ & $671(42.2 \%)$ & $146(39.9 \%)$ & $525(43.0 \%)$ & \\
\hline $5-6$ & $38(2.4 \%)$ & $10(2.7 \%)$ & $28(2.3 \%)$ & \\
\hline EMT level & & & & 0.027 \\
\hline Intermediate & $770(48.5 \%)$ & $196(53.6 \%)$ & $574(47.0 \%)$ & \\
\hline Paramedic & $818(51.5 \%)$ & $170(46.4 \%)$ & $648(53.0 \%)$ & \\
\hline \multicolumn{5}{|l|}{ Type of life support } \\
\hline ALS & $811(51.1 \%)$ & $194(53.0 \%)$ & $617(50.5 \%)$ & 0.399 \\
\hline BLS & $777(48.9 \%)$ & $172(47.0 \%)$ & $605(49.5 \%)$ & \\
\hline \multicolumn{5}{|l|}{ EMS parameter, min } \\
\hline response time, mean (SD) & $6.07(2.9)$ & $5.5(3.03)$ & $6.21(2.87)$ & 0.832 \\
\hline scene time interval, mean (SD) & $13.51(5.03)$ & $13.29(4.60)$ & $13.57(5.15)$ & 0.099 \\
\hline transport time, mean (SD) & $5.22(3.64)$ & $4.14(2.84)$ & $5.53(3.79)$ & $<0.001$ \\
\hline Alive over $2 \mathrm{~h}$ & $624(39.3 \%)$ & $113(30.9 \%)$ & $511(41.8 \%)$ & $<0.001$ \\
\hline Admitted to ICU & $571(36.0 \%)$ & $106(29.0 \%)$ & $465(38.1 \%)$ & 0.001 \\
\hline Survival at discharge & $287(18.1 \%)$ & $39(10.7 \%)$ & $248(20.3 \%)$ & $<0.001$ \\
\hline $\mathrm{CPC}$ & & & & $<0.001$ \\
\hline 1 & $121(7.6 \%)$ & $13(3.6 \%)$ & $108(8.8 \%)$ & \\
\hline 2 & $57(3.6 \%)$ & $5(1.4 \%)$ & $52(4.3 \%)$ & \\
\hline 3 & $58(3.7 \%)$ & $9(2.5 \%)$ & $49(4.0 \%)$ & \\
\hline 4 & $51(3.2 \%)$ & $12(3.3 \%)$ & $39(3.2 \%)$ & \\
\hline 5 & $1031(81.9 \%)$ & $327(89.3 \%)$ & $974(79.7 \%)$ & \\
\hline \multicolumn{5}{|l|}{ Post-cardiac arrest care } \\
\hline Emergency PCI & & & $332(27.2 \%)$ & \\
\hline TTM & & & $412(33.7 \%)$ & \\
\hline
\end{tabular}

Values are expressed as number (\%) unless otherwise specified. SD, standard deviation; CPR, cardiopulmonary resuscitation; EMT, emergency medical technician; BLS, basic life support; ALS, advanced life support; ICU, intensive care unit; $C P C$, cerebral performance category.

Table 2. Patients delivered to Heart Centers compared to nonHeart Centers had better survival over 2 hours (aOR, 1.40; 95\% confidence interval [CI], 1.14-1.72), favorable admission to the ICU (aOR, 1.36; 95\% CI, 1.10-1.69), better survival at discharge (aOR, 2.00; 95\% CI, 1.42-2.81), and favorable CPC score (aOR, 3.14; 95\% CI, 1.88-5.23). Longer transport time was related to unfavorable survival outcomes including survival over 2 hours (aOR, 0.73; 95\% CI, 0.55-0.97) and admission to the ICU (aOR, 0.69; 95\% CI, 0.51-0.92), but transport time had no significant difference in survival at discharge and favorable CPC score.

The survival analysis shows the HRs of the multivariable Cox regression model (Table 2 ). The overall mortality reduction for Heart Center was 39\% $(\mathrm{HR}=0.61 ; 95 \% \mathrm{CI}, 0.47-0.78)$ compared to that for the non-Heart Center. Longer transport time increased the risk of mortality by $19 \%(\mathrm{HR}=1.19 ; 95 \%$ 
TA B L E 2. Risk prediction of survival outcomes in OHCA patient with shockable rhythm.

\begin{tabular}{|c|c|c|c|c|}
\hline Outcomes & Factors & OR $(95 \% \mathrm{CI})$ & $\mathrm{aOR} * *(95 \% \mathrm{CI})$ & $\mathrm{aOR} * * *(95 \% \mathrm{CI})$ \\
\hline \multicolumn{5}{|c|}{ Alive over $2 \mathrm{~h}$} \\
\hline & Heart Center (Y vs. N) & $1.69(1.25-2.07)$ & $1.78(1.37-2.30)$ & $1.40(1.14-1.72)$ \\
\hline & Transport time (long vs. short) & $0.76(0.59-0.99)$ & $0.79(0.60-1.04)$ & $0.73(0.55-0.97)$ \\
\hline \multicolumn{5}{|c|}{ Admitted to ICU } \\
\hline & Heart Center (Y vs. N) & $1.51(1.17-1.94)$ & $1.67(1.29-2.18)$ & $1.36(1.10-1.69)$ \\
\hline & Transport time (long vs. short) & $0.75(0.58-0.99)$ & $0.80(0.61-1.07)$ & $0.69(0.51-0.92)$ \\
\hline \multicolumn{5}{|c|}{ Survival at discharge } \\
\hline & Heart Center (Y vs. N) & $2.14(1.49-3.06)$ & $2.48(1.70-3.63)$ & $2.00(1.42-2.81)$ \\
\hline & Transport time (long vs. short) & $0.77(0.55-1.09)$ & $0.83(0.57-1.20)$ & $0.78(0.57-1.07)$ \\
\hline \multicolumn{5}{|l|}{ Good CPC } \\
\hline & Heart Center (Y vs. N) & $2.91(1.76-4.81)$ & $3.50(2.07-5.91)$ & $3.14(1.88-5.23)$ \\
\hline & Transport time (long vs. short) & $0.92(0.61-1.38)$ & $0.99(0.64-1.54)$ & $0.90(0.59-1.39)$ \\
\hline \multicolumn{5}{|l|}{ Death $^{\dagger}$} \\
\hline & Heart Center (Y vs. N) & & & $0.61(0.47-0.78)$ \\
\hline & Transport time (long vs. short) & & & $1.19(0.90-1.58)$ \\
\hline
\end{tabular}

* p-value < 0.05; aOR, adjusted odds ratio; ICU, intensive care unit; CPC, cerebral performance category; $C I$, confidence interval.

** aOR (adjusted odds ratio): adjusted by age, sex, location of arrest, witness of collapse, provision of bystander, shift of non-shock rhythm, number of EMTs, type of life support, response time, and Scene time interval.

*** PS-adjust (quintiles).

$\dagger$ analyzed by cox PH model, with HR (hazard ratio) and 95\% CI in the cells.

CI, 0.90-1.58).

The Kaplan-Meier survival curve displayed the 120-day cumulative survival rate by comparing patients who were transported to the non-Heart or Heart Center within a long and short time (Fig. 2). The log-rank test results showed a higher survival rate in patients who were transported to Heart Centers within a short time after follow-up for 120 days $(p<0.001)$. In Fig. 3, the percentage of good CPC (defined as less than 2) was $16.3 \%$ in patients transported to non-Heart Centers within a short time, $11.1 \%$ in those transported to non-Heart Centers within a long time, $31.0 \%$ in those transported to Heart Centers within a short time, $32.6 \%$ in those transported to Heart Centers within a long time.

\section{Discussion}

This present study particularly focusses on the fourth and fifth parts of the survival chain, which are direct transport to the Heart Center and survival curve after discharge Our study found that OHCA patients with initial shockable rhythm and without prehospital ROSC directly delivered to the Heart Center have a higher probability of four months survival, survival $[\mathrm{aOR}=2.00]$ and favorable neurological outcomes $[\mathrm{aOR}=3.14]$ at discharge, but long transport time did not cause poor survival or neurological outcomes in multiple conditional logistic regression models. We also analyzed these patients who survived after 2 hours and found better neurological outcomes if they were transported to the Heart Center. However, the survival Cox analysis showed an increased risk for these patients who had a longer transport time (over 7.16 minutes)

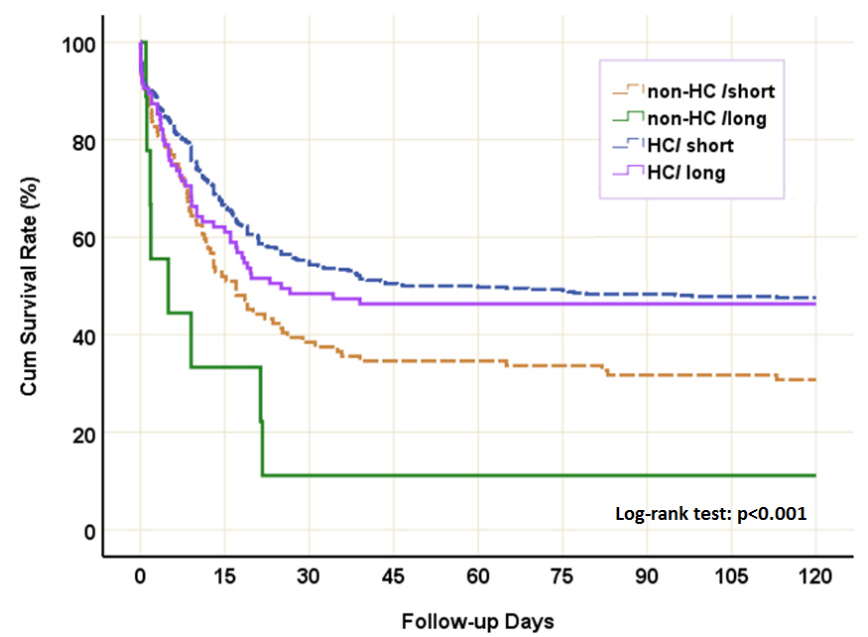

F I G URE 2. Kaplan Meier survival curves of patients who survived after two hours displays four combinations of transporting to Heart Centers. Pairwise log rank tests showed the difference between non-HC/short and $\mathrm{HC} /$ short ( $p=0.001)$, between non-HC/long and HC/short $(p=0.001)$, between non-HC/long and HC/long $(p=0.011)$, between non$\mathrm{HC} /$ short and $\mathrm{HC} /$ long $(p=0.048)$, between non-HC/long and non-HC/short $(p=0.049)$.

Abbreviations: HC, Heart Centers.

to the Heart Center would have poor survival $(\mathrm{HR}=1.19)$, but this was not significant in the model. These results were also consistent with those of a previous study on survival and good 


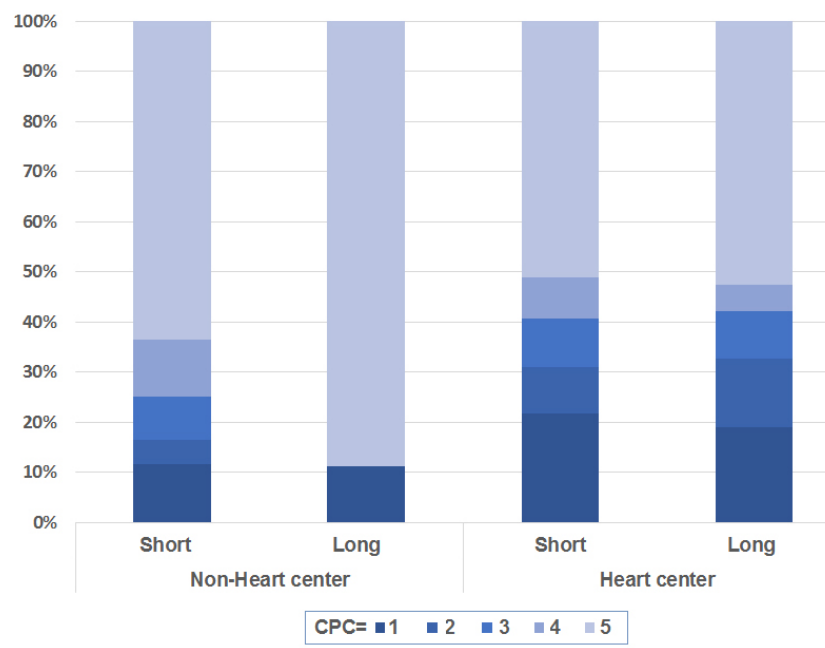

F I G URE 3. Bar chart of CPC distribution of patients who survived after two hours grouped by four combinations of transporting to Heart Centers. The percentages of good CPC (defined less than 2) are $16.3 \%$ in non-HC/short, $11.1 \%$ in non-HC/long, $31.0 \%$ in $\mathrm{HC} /$ short, $32.6 \%$ in $\mathrm{HC} /$ long. $p<0.001$ by Chi-square test.

Abbreviations: CPC, Cerebral Performance Category.

neurological outcomes if EMTs delivered OHCA patients with initial shockable rhythm to the Heart Center [19-21].

Previous studies suggested that the majority of OHCA patients who needed primary PCIs likely had coronary artery disease. In addition, shockable rhythm also needed PCIs to rule out coronary artery disease $[17,19]$. According to our findings, OHCA patients with initial shockable rhythm and without prehospital ROSC who were delivered to the hospital with the capability of receiving PCIs, which was defined as Heart Centers even under longer transport time, could have better survival and neurological outcomes [22-24].

Regarding the characteristics of patients of our study population, patients delivered to Heart Centers with a trend to be more witness of collapse. In previous studies, when witnessed by either EMS or a bystander, the survival rate of OHCA patients seemed higher [6]. Tsai et al. [23] reported that witnessed collapse was an independent predictor of both survival at discharge and good CPC (OR $=2.23$ and 3.52, respectively). Excessive transport time may affect survival in patients who are alive over 2 hours and alive in the ICU, but it is not significant in the secondary outcome. Considering the above results and those of previous studies, EMTs take adequate time to transport OHCA patients with initial shockable rhythm and without prehospital ROSC to Heart Centers, and this may help achieve better survival or neurological outcomes [25]. Unlike the previous study, we used propensity score stratification to control for bias and confounding factors due to the limitation of the observation study.

Previous studies have emphasized direct transport to PCIcapable hospitals, and transport time affects OHCA patients' outcomes separately. Similar to our study, Nicole reported good survival outcomes $(\mathrm{OR}=1.97)$ if patients were directly delivered to PCI-capable hospitals within 47 minutes from the EMS call to arrive at the hospital [17].
It is known that OHCA patients with initial shockable rhythm have better outcomes or less neurologic deficit than those with non-shockable rhythm [24]. Past studies included the OHCA population including shockable rhythm or non-shockable rhythm. Our study focuses on shockable rhythm in OHCA patients because those patients had a high probability of cardiac etiology $(70 \%-80 \%)$ and needed more integrated post-cardiac arrest care [26]. Kragholm et al. [19] found that direct transport to PCI centers is associated with better survival rates and neurological outcomes in OHCA patients, even when bypassing the nearest hospital, although the transport time was longer than 30 minutes. Low transport time did not result in better survival or neurological outcomes during discharge in adult OHCA patients. In line with previous findings, conditional multivariable logistic regression performed in our study suggested that there is no difference in survival or neurological outcomes except for those alive in the ICU. Elmer et al. [20] also found that direct transport to cardiac arrest patients-receiving centers from the scene is independently associated with reduced mortality $[27,28]$.

However, survival with a 4-month follow-up may be decreased if shockable OHCA patients have long transport time delivery to non-Heart Centers as noted in our Kaplan-Meier curve. Gordon suggested that, if necessary, the delivery of OHCA patients to Heart Centers by the EMS should not exceed 15 minutes [13]. Our results showed enough benefit for direct transport to Heart Centers, but we wasted much transport time; this could increase pressure on local EMS systems, such as increasing occupied ambulance and EMTs. It is important for the emergency medical system to evaluate the appropriate delivery distance and time to avoid the loss of emergency resources. Hence, this implied that OHCA patients with shockable rhythm should be delivered directly to Heart Centers in adequate transport time.

Other studies included OHCA patients with prehospital ROSC, which is different from our study. Prehospital ROSC may indicate that those patients initially had better outcomes and better performance in the first to third chains of survival, such as high-performance CPR, good chest compression fraction, or short duration of the first shock. We focused on populations of OHCA patients without pre-hospital ROSC to evaluate the ability or level of handling cardiac arrest patients after arriving at the hospital. The results were consistent with those of Kajino et al. [28], who revealed that OHCA patients without prehospital ROSC who were transferred to Heart Centers will have higher odds of survival at discharge and neurologic outcomes due to integrated post-cardiac arrest care in Heart Centers.

\section{Limitation}

Our study is limited due to its retrospective and observational nature. Although the characteristics of the population are very similar in both groups, statistical bias may have been introduced by the limited local population and selection bias, even though we used propensity score stratification. Other information such as underlying diseases and cause(s) of death were not collected and analyzed because of limited in-hospital 
data. The results of the present study may have some consideration if applied to other countries due to diffident geography, EMS distribution, and distance to Heart Centers or non-Heart Centers. Hence, further studies should assess whether or not OHCA patients with initial shockable rhythm should be randomly transported to Heart Centers to evaluate the effect of Heart Centers in the future.

\section{Conclusions}

Adult OHCA patients with initial shockable rhythm without prehospital ROSC who were directly transported to Heart Centers had better chances of four months survival and good neurologic outcomes, regardless of the transport time.

\section{AUTHOR CONTRIBUTIONS}

Conceptualization, CCY, CHC, YMW, CCL, CJN, CJS, $\mathrm{CHH}$, LHT; Data curation, CBC; Formal analysis, CBC, HJT; Investigation, CJN, YMW; Methodology, LHT, CCL and CHH; Software, HJT; Supervision, CJS; Writing - original draft, CCY and CHC; Writing - review \& editing, LHT. CCY and $\mathrm{CHC}$ contributed equally to this work.

\section{ETHICS APPROVAL AND CONSENT TO PARTICIPATE}

This study was approved by the Hospital Ethics Committee on Human Research of the Chang Gung Medical Foundation (Taiwan, ROC; IRB Permit No.: 201600635B0).

\section{ACKNOWLEDGMENT}

We would like to thank Editage (www. editage.com) for English language editing.

\section{FUNDING}

This research received no external funding.

\section{CONFLICT OF INTEREST}

The authors declare no conflict of interest.

\section{SUPPLEMENTARY MATERIAL}

Supplementary material associated with this article can be found, in the online version, at https://oss. signavitae. com/mre-signavitae/article/1391680702544527360/ attachment/SV2021031201_supplementary1.tif.

\section{REFERENCES}

[1] Nichol G, Thomas E, Callaway CW, Hedges J, Powell JL, Aufderheide $\mathrm{TP}$, et al. Regional variation in out-of-hospital cardiac arrest incidence and outcome. Journal of the American Medical Association. 2008; 300: 1423-1431.

[2] Hasegawa K, Tsugawa Y, Camargo CA Jr, Hiraide A, Brown DFM. Regional variability in survival outcomes of out-of-hospital cardiac arrest: the all-Japan Utstein Registry. Resuscitation. 2013; 84: 10991107.

[3] Hu S. Out-of-hospital cardiac arrest in an oriental metropolitan city. The American Journal of Emergency Medicine. 1994; 12: 491-494.

[4] Sulzgruber P, Sterz F, Schober A, Uray T, Van Tulder R, Hubner P, et al. Editor's Choice-Progress in the chain of survival and its impact on outcomes of patients admitted to a specialized high-volume cardiac arrest center during the past two decades. European Heart Journal: Acute Cardiovascular Care. 2016; 5: 3-12.

[5] Cummins RO. Emergency medical services and sudden cardiac arrest: the "chain of survival" concept. Annual Review of Public Health. 1993; 14: 313-333.

[6] Cummins RO, Ornato JP, Thies WH, Pepe PE. Improving survival from sudden cardiac arrest: the "chain of survival" concept. A statement for health professionals from the Advanced Cardiac Life Support Subcommittee and the Emergency Cardiac Care Committee, American Heart Association. Circulation. 1991; 83: 1832-1847.

[7] Chien C, Fang S, Tsai L, Tsai S, Chen C, Seak C, et al. Traditional versus blended CPR training program: a randomized controlled non-inferiority study. Scientific Reports. 2020; 10: 10032.

[8] Wang M, Wu Y, Chien C, Tsai L, Chen C, Seak C, et al. Learning effectiveness assessment between primary school students and adults in basic life support education. Emergency Medicine International. 2021; 2021: 5579402.

[9] Sasson C, Rogers MAM, Dahl J, Kellermann AL. Predictors of survival from out-of-hospital cardiac arrest. Circulation: Cardiovascular Quality and Outcomes. 2010; 3: 63-81.

${ }^{[10]}$ Li Q, Goodman SG, Yan RT, Gore JM, Polasek P, Lai K, et al. Prehospital cardiac arrest in acute coronary syndromes: insights from the global registry of acute coronary events and the Canadian registry of acute coronary events. Cardiology. 2013; 126: 27-34.

[11] Lin Y, Chang S, Wang L, Chi H, Ueng K, Tsai C, et al. Prehospital predictors of initial shockable rhythm in out-of-hospital cardiac arrest: findings from the Taichung Sudden Unexpected Death Registry (THUNDER). Mayo Clinic Proceedings. 2017; 92: 347-359.

[12] Martínez-Losas P, Salinas P, Ferrera C, Nogales-Romo MT, Noriega F, Trigo MD, et al. Coronary angiography findings in cardiac arrest patients with non-diagnostic post-resuscitation electrocardiogram: a comparison of shockable and non-shockable initial rhythms. World Journal of Cardiology. 2017; 9: 702-709.

[13] Ewy GA, Bobrow BJ. Cardiocerebral resuscitation: an approach to improving survival of patients with primary cardiac arrest. Journal of Intensive Care Medicine. 2016; 31: 24-33.

[14] Nolan JP, Soar J, Cariou A, Cronberg T, Moulaert VRM, Deakin CD, et al. European resuscitation council and european society of intensive care medicine guidelines for post-resuscitation care 2015: section 5 of the European Resuscitation Council Guidelines for Resuscitation 2015. Resuscitation. 2015; 95: 202-222.

[15] Schober A, Sterz F, Laggner AN, Poppe M, Sulzgruber P, Lobmeyr E, et al. Admission of out-of-hospital cardiac arrest victims to a high volume cardiac arrest center is linked to improved outcome. Resuscitation. 2016; 106: 42-48.

[16] Søholm H, Kjaergaard J, Bro-Jeppesen J, Hartvig-Thomsen J, Lippert F, Køber L, et al. Prognostic implications of level-of-care at tertiary heart centers compared with other hospitals after resuscitation from out-of-hospital cardiac arrest. Circulation: Cardiovascular Quality and Outcomes. 2015; 8: 268-276.

[17] McKenzie N, Williams TA, Ho KM, Inoue M, Bailey P, Celenza A, et al. Direct transport to a PCI-capable hospital is associated with improved survival after adult out-of-hospital cardiac arrest of medical aetiology. Resuscitation. 2018; 128: 76-82.

${ }^{[18]}$ Chien C, Tsai S, Tsai L, Chen C, Seak C, Weng Y, et al. Impact of transport time and cardiac arrest centers on the neurological outcome after out-of-hospital cardiac arrest: a retrospective cohort study. Journal of the American Heart Association. 2020; 9: e015544.

[19] Kragholm K, Malta Hansen C, Dupre ME, Xian Y, Strauss B, Tyson $\mathrm{C}$, et al. Direct transport to a percutaneous cardiac intervention center and outcomes in patients with out-of-hospital cardiac arrest. Circulation: Cardiovascular Quality and Outcomes. 2017; 10: e003414. 
[20] Elmer J, Callaway CW, Chang CH, Madaras J, Martin-Gill C, Nawrocki $\mathrm{P}$, et al. Long-term outcomes of out-of-hospital cardiac arrest care at regionalized centers. Annals of Emergency Medicine. 2019; 73: 29-39.

[21] Han KS, Lee SW, Lee EJ, Kim SJ. Prognostic value of the conversion to a shockable rhythm in out-of-hospital cardiac arrest patients with initial non-shockable rhythm. Journal of Clinical Medicine. 2019; 8: 644.

[22] Geri G, Gilgan J, Wu W, Vijendira S, Ziegler C, Drennan IR, et al. Does transport time of out-of-hospital cardiac arrest patients matter? A systematic review and meta-analysis. Resuscitation. 2017; 115: 96-101.

[23] Tsai S, Chaou C, Huang C, Tzeng I, Kuo C, Weng Y, et al. Features of hospital and emergency medical service in out-of-hospital cardiac arrest patients with shockable rhythm. The American Journal of Emergency Medicine. 2017; 35: 1222-1227.

[24] Vyas A, Chan PS, Cram P, Nallamothu BK, McNally B, Girotra S. Early coronary angiography and survival after out-of-hospital cardiac arrest. Circulation: Cardiovascular Interventions. 2015; 8: e002321.

[25] Garcia S, Drexel T, Bekwelem W, Raveendran G, Caldwell E, Hodgson $\mathrm{L}$, et al. Early access to the cardiac catheterization laboratory for patients resuscitated from cardiac arrest due to a shockable rhythm: the minnesota resuscitation consortium twin cities unified protocol. Journal of the
American Heart Association. 2016; 5: e002670.

[26] Kern KB, Lotun K, Patel N, Mooney MR, Hollenbeck RD, McPherson $\mathrm{JA}$, et al. Outcomes of comatose cardiac arrest survivors with and without ST-segment elevation myocardial infarction: importance of coronary angiography. JACC: Cardiovascular Interventions. 2015; 8: 1031-1040.

[27] Edgren E, Hedstrand U, Kelsey S, Sutton-Tyrrell K, Safar P. Assessment of neurological prognosis in comatose survivors of cardiac arrest. BRCT I Study Group. Lancet. 1994; 343: 1055-1059.

[28] Kajino K, Iwami T, Daya M, Nishiuchi T, Hayashi Y, Kitamura T, et al. Impact of transport to critical care medical centers on outcomes after outof-hospital cardiac arrest. Resuscitation. 2010; 81: 549-554.

How to cite this article: Chung-Cheng Yeh, Chia-Hau Chang, Chen-June Seak, Chen-Bin Chen, Yi-Ming Weng, Chi-Chun Lin, et al. Survival analysis in out-of-hospital cardiac arrest patients with shockable rhythm directly transport to Heart Centers. Signa Vitae. 2021;17(5):95-102. doi:10.22514/sv.2021.084. 